\title{
INDICADORES BIBLIOMÉTRICOS SOBRE EL PLAN DE ACCIÓN TUTORIAL EN LA UNIVERSIDAD PARA EL ALUMNADO CON DISCAPACIDAD DESDE 1988-2018
}

\author{
Gonzalo Lorenzo Lledó \\ glledo@ua.es \\ Asunción Lledó Carreres \\ asuncion.lledo@gcloud.ua.es \\ Alejandro Lorenzo Lledó \\ alejandro.Iorenzo@ua.es \\ Graciela Arráez Vera \\ Graciela.arraez@ua.es \\ Marcos Gómez-Puerta \\ Marcos.gomez@ua.es \\ Facultad de Educación. \\ Universidad de Alicante
}

Fecha de Recepción: 9 Marzo 2018

Fecha de Admisión: 10 Abril 2018

\section{RESUMEN}

La diversidad presente en las aulas universitarias es un elemento al que tiene que dar respuesta el profesorado. Existen multitud de herramientas con los que dar respuesta a la diversidad funcional y una de ellas es la acción tutorial. El objetivo de la investigación es realizar un análisis de la producción científica sobre la acción tutorial en la Universidad para el alumnado con discapacidad. Para ello se utiliza la ISI Web of Science y estableciendo como términos de búsqueda acción tutorial, discapacidad y plan de acción tutorial; en castellano y en inglés. Se obtiene una muestra de 78 artículos que se estudiarán a partir de unos indicadores bibliométricos como por ejemplo las áreas de investigación, categorías de la web of Science, años de publicación. Los resultados indican que el periodo de 2015-2016 fue el año de mayor producción científica, siendo el inglés el idioma de mayor producción científica. A pesar de ello España es el mayor productor sobre la temática; destacando la Universidad de Granada y Burgos como los centros más destacados. Finalmente Education \& Educational Research es la categoría y el área de investigación donde se generan más artículos. A pesar de ello las áreas tecnológicas están adquiriendo un mayor peso debido a la versatilidad que ofrece la tecnología. Como futuras líneas de investigación se plantea la posibilidad de analizar los contenidos estudiados y aquellas áreas educativas más trabajadas.

Palabras clave: plan de acción tutorial; discapacidad, bibliométrico, respuesta educativa 


\section{ABSTRACT \\ Bibliometric indicators on the tutorial action plan at the university for students with disabili- ties from 1988-2018"}

The diversity present in university classrooms is an element that teachers are founding. There are many tools to respond to functional diversity and one of them is the tutorial action. The objective of this research is to analyze the scientific production of the tutorial action in the University for students with disabilities. For this purpose, the ISI Web of Science is used and the search terms tutorial action, disability and tutorial action plan are established; in Spanish and English. A sample of 78 articles is obtained and will be studied based on bibliometric indicators such as research areas, categories of the Web of Science, years of publication. The results indicate that the period 20152016 produced the vast majority of the papers. Furthermore, English is the language of greatest scientific production. In spite of this, Spain is the largest producer on the subject, with the University of Granada and Burgos as the most outstanding centres. Finally, Education \& Educational Research is the category and area of research where most articles are generated. However, the technological areas are gaining in importance due to the versatility of the technology. As future lines of research, the possibility of analysing the contents studied and the most studied educational areas is proposed.

Keywords: tutorial action plan; disability; university; educational response

\section{ANTECEDENTES}

Las sociedades actuales están insertadas en una vorágine de continuo cambio y transformación dando una continua respuesta a las necesidades de sus ciudadanos. A lo largo de este desarrollo continuo la Educación tiene un papel fundamental. Es necesaria la inclusión de todos aquellos núcleos de población más desfavorecidos y especialmente el alumnado con discapacidad. Este alumnado ha sido excluido sistemáticamente de todos los niveles educativos a lo largo de la historia. Pero las diversas leyes educativas Españolas han ido permitiendo una mejora de sus derechos y especialmente conseguir su llegada al escalafón más alto del sistema educativo la Educación Superior Universitaria.

En esta misma línea el Informe Universidad Bricall (2000) expresó en sus investigaciones la importancia del profesor tutor del estudiante universitario. De esta forma se podrían dar respuestas las necesidades que pudiera presentar el alumnado. A partir de esta línea de trabajo diferentes estudios como los de Álvarez \& Rodríguez (2000); Lázaro (2002); Rodríguez (2006); Álvarez (2009,2013); Pérez (2006); García (2008); Álvarez (2010) han puesto de manifiesto la importancia de la acción tutorial y el Plan de Acción tutorial en la Universidad como pieza de vital importancia en la formación del alumnado. Posteriormente Rodríguez (2004) afirmaba la existencia de dos modelos para llevar a cabo la función tutorial del profesorado: el modelo académico y el modelo profesional. En el primero de los modelos Rodríguez (2004) concluía que estaba basado en la información que necesitaba el alumnado para poder aprobar una asignatura. Por el contrario en el modelo profesional; Rodríguez (2004) afirmaba que tenía como objetivo cubrir las necesidades que se generaban en cuanto al desarrollo profesional del alumno. Lledó et al. (2008) intenta mejorar el modelo de tutor descrito por Rodríguez (2004) proponiendo un nuevo perfil de docente/tutor universitario. Por un lado Lledó et al (2008) planteaba que el docente recabara información sobre el seguimiento del alumno de forma que se produzca una mejora de su rendimiento y permitiera una intervención formativa para dar respuesta a sus necesidades. En contraposición y siguiendo la línea de Rodríguez (2004); se expone la necesidad de una orientación mixta académica y profesional para que el alumnado pueda conseguir su proyecto personal. Para Álvarez (2008), la acción tutorial proporciona un elemento de calidad a la educación superior. Para estos autores esto se debe a la capacidad de pro- 
ducir tareas de información y orientación que puedan implicar a todos los elementos que forman parte del proceso de aprendizaje. En la misma línea, Perandones \& Lledó (2009) obtienen como resultado en su investigación que la acción tutorial está destinada a ayudar la planificación del desarrollo académico, personal y profesional del alumnado. Recientemente Álvarez (2014) afirmaba que el PAT permite detectar necesidades, solapamientos relacionados con planes de estudios además de las carencias que muestra el alumnado en su desarrollo personal, social y profesional además de la detección de los colectivos de riesgo de abandono.

Como apunta Lledó (2015) el PAT tiene que conseguir que los entornos educativos caracterizados como discapacitantes puedan ser convertidos en inclusivos Esta respuesta surge como consecuencia de la presencia de la discapacidad en la universidad. Para Lorenzo, Lledó, Arráez \& Lorenzo (2017) la Universidad y más concretamente el PAT debe vencer las barreras provocadas por el desconocimiento de la normativa actual; además de la no existencia de la regulación en cuanto a las medidas de adaptación de este alumnado. Esta afirmación parte de los resultados indicados por Moriña, Díez \& Molina Romo (2011) que indicaban que el profesorado demandaba una mayor formación sobre la atención a la diversidad y las adaptaciones curriculares asociadas. Siendo el PAT el elemento de inclusión de esta información tanto para el profesorado para el alumnado.

A partir de la revisión teórica realizada se plantea la necesidad de analizar cuál ha sido la evolución de la producción científica sobre la acción tutorial y el alumnado con discapacidad en la Universidad. Para ello se utilizarán una serie de indicadores que permitirán obtener la información sobre aquellas áreas; idiomas; años de publicación categorías de la Web of Science... que definirán el estado actual de la investigación.

\section{OBJETIVOS}

Tomando como referencia la justificación teórica presentada en el apartado anterior, se plantea como objetivo general realizar un análisis bibliométrico sobre la producción científica existente sobre el Plan de Acción Tutorial en el alumnado con discapacidad en el entorno universitario el periodo 1988-2018. Para ello se establecen dos objetivos más específicos.

¿Son los indicadores bibliométricos presentes en el estudio una herramienta de clasificación de la información?

¿Es el tópico de estudio un tema con suficiente proyección investigadora?

\section{MUESTRA DE PARTICIPANTES}

La muestra de unidades de análisis se ha conformado por 78 artículos publicados sin ningún tipo de restricción temporal y fueron obtenidos de la base de datos principal de la Web of Science. Se llevó a cabo un estudio de búsqueda, selección y lectura individual y análisis de los datos desde septiembre 2017 hasta diciembre 2017.

\section{METODOLOGÍA E INSTRUMENTO}

Teniendo como punto de partida el objetivo del estudio se ha llevado a cabo un diseño bibliométrico descriptivo-restrospectivo lo que-según (Montero \& Leon, 2007) permite seleccionar y organizar los documentos. Como consecuencia, los pasos a seguir son los siguientes: en primer lugar, búsqueda y selección de la información; en segundo lugar, clasificación (según los descriptores) de los documentos hallados; y finalmente, análisis de los datos (Rosa et al. 1996).

Para realizar la búsqueda se utilizó la ISI Web of Science y en su buscador se introdujeron los siguientes términos tanto en castellano como en inglés:

Tema: tutorial action or tema: plan de acción tutorial and disability and tema: university and PAT or acción tutorial. Se escogieron que dichos términos estuvieran presentes tanto en el título como 
en el tema mediante el termino AND. Tras realizar este paso se selección como base de datos donde buscar los artículos la colección principal de la Web of Science. Dentro de todos los indicadores que tiene esta base fueron descartados los libros (Book Citation Index). En cuanto al periodo de tiempo para realizar la búsqueda no se le puso limitación. Luego de realizar estos ajustes se obtuvo una muestra de 78 artículos. Una vez obtenida la cantidad de documentos deseada, se pasó a examinar los mismos. Se siguieron los siguientes pasos: 1) seleccionar y organizar los documentos relevantes para la investigación y 2) establecer una serie de variables necesarias para la catalogación de la información (tabla 1):

\section{Tabla 1}

Selección de variables en la clasificación de investigaciones sobre PAT y discapacidad.

\begin{tabular}{|c|c|}
\hline Variables & Descripción \\
\hline Documento & Título, año de publicación y ámbito de investigación \\
\hline Autoría & $\mathrm{N}^{\mathrm{o}}$ de autor/es que lo realizan, e institución a la que pertenecen \\
\hline Revista & Título, temática, base de datos en la que se encuentra, valor de impacto \\
\hline Idioma & Español/Inglés \\
\hline País & País \\
\hline
\end{tabular}

Tras obtener la muestra final (78 documentos), se establecieron una serie de indicadores bibliométricos, los cuales permitieron llevar a cabo el posterior análisis (tabla 2):

Tabla 2

Indicadores bibliométricos para el análisis del tópico de investigación.

\begin{tabular}{|c|c|}
\hline Indicadores bibliométricos & Descripción \\
\hline $\begin{array}{l}\text { 1.- Numero de citas por año de } \\
\text { publicación }\end{array}$ & $\begin{array}{l}\text { Cantidad de citas anuales que reciben los } \\
\text { artículos durante el periodo estudiado. }\end{array}$ \\
\hline 2-Año de publicación & $\begin{array}{c}\text { Cantidad de documentos anuales que reciben los } \\
\text { artículos durante el periodo estudiado. }\end{array}$ \\
\hline 3-Idiomas & Idiomas en los que se publican los documentos \\
\hline 4-Países productores & $\begin{array}{c}\text { Los países productores sobre el tópico de la } \\
\text { temática. }\end{array}$ \\
\hline 5-Tipos de documentos & $\begin{array}{l}\text { Los diferentes formatos que presentan los } \\
\text { documentos publicados sobre el tópico de } \\
\text { estudio. }\end{array}$ \\
\hline 6-Categorías de la Web of Science & $\begin{array}{l}\text { Cantidad de documentos según las diferentes } \\
\text { categorías que establece la Web of Science }\end{array}$ \\
\hline 7-Areas de investigación & $\begin{array}{c}\text { Áreas de investigación más productivas sobre } \\
\text { PAT }\end{array}$ \\
\hline 8.- Autores productores & Autores más productores \\
\hline 9.- Coautoría. & Cantidad de autores por trabajo realizado \\
\hline
\end{tabular}




\section{RESULTADOS}

A continuación se detallan los resultados obtenidos a partir de las variables establecidas en el estudio:

Número de citas por año de publicación.

El primero de los indicadores es la evolución temporal del número de citas con respecto al año de publicación. Tras analizar la figura 1 se puede concluir que a partir del año 2013 se produce un incremento del número de citas; alcanzando su punto máximo en 2015 con 12 citas. Tras un breve descenso en 2016, a partir de 2017 se produce otro ligero incremento llegando a las 10 citas. A lo largo de este año 2018, solo han recibido 1 cita.

Figura 1. Evolución del número de citas por años.

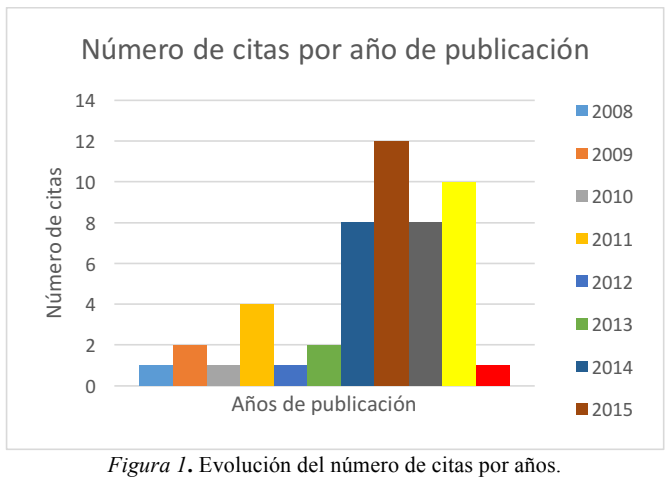

Porcentaje de artículos publicados por año de publicación.

La segunda variable del estudio es el porcentaje de artículos publicados por año de publicación; esta información está representada en la figura 2. Aquellos años que presentan una mayor producción científica son 2015 con un 23\% de los artículos publicados, seguido por el 2010,2011, 2017 que han realizado el $12 \%$ de las publicaciones. Uno de los años menos prolijos fue 1988 con solo el $1 \%$ de los artículos.

Figura 2. Porcentaje de artículos por año de publicación.

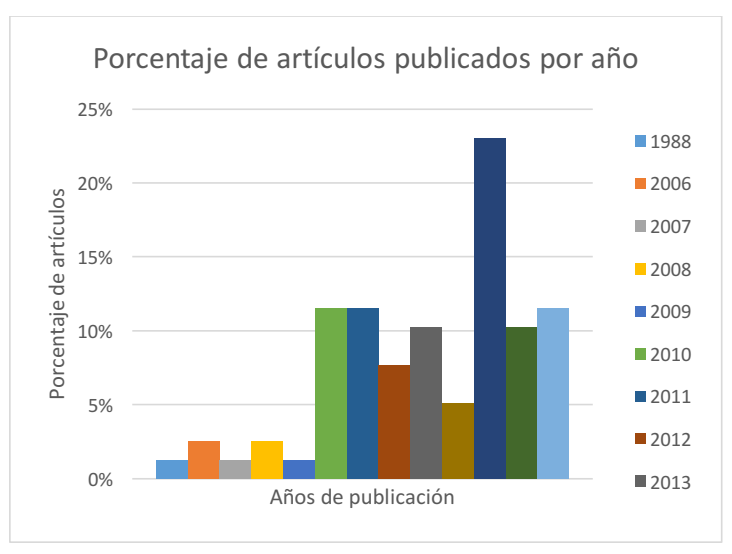


Idioma de publicación

Al realizar el análisis de cualquier temática de investigación es muy importante profundizar en aquellas lenguas donde se produce la mayor difusión de los artículos. Esta información queda reflejada en la figura 3. Siendo el idioma predominante el inglés con un $68 \%$ de las publicaciones de carácter científica seguido del castellano con el $31 \%$ de los escritos. El tercero de los idiomas es el portugués con el $1 \%$ de las publicaciones.

Figura 3. Porcentaje de artículos por idiomas

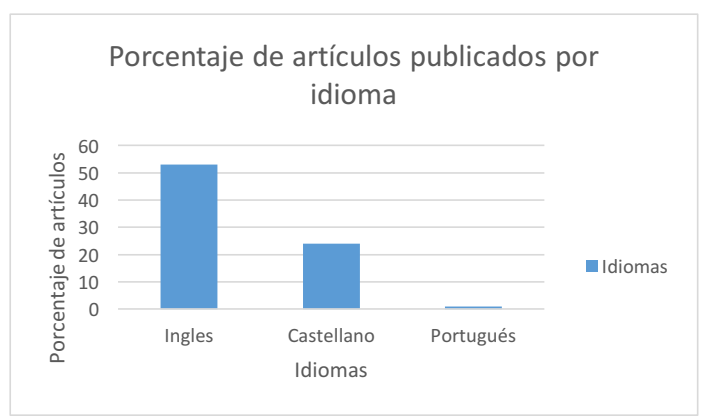

Países productores

La cuarta de las variables que se analiza a lo largo del estudio son los países donde mayor producción científica existe sobre la temática. En el caso de España es donde más artículos se producen con casi el 90\%, seguido de Méjico con solo el 8\% de los artículos. Por el contrario en Inglaterra solo se han producido el $1 \%$ de las investigaciones.

Figura 4. Porcentaje de artículos por país.

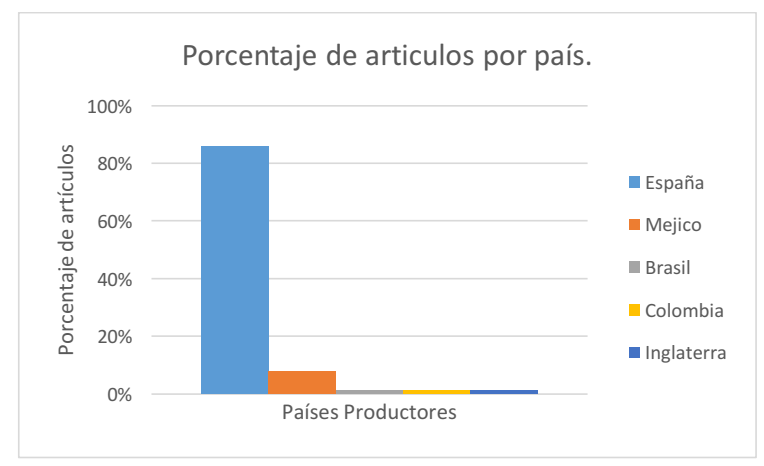

Tipos de documentos

Para llevar a cabo un correcto análisis de la literatura científica se hace necesario el determina el tipo de documento con el que se está trabajando. La figura 5 es una representación de los diversos tipos de documentos que existen en la literatura consultada. Se puede observar como el $65 \%$ de las publicaciones corresponden a congresos, siendo solo el $33 \%$ referida a investigaciones publicadas en revistas especializadas. 
Figura 5. Porcentaje de artículos por tipo de documento.

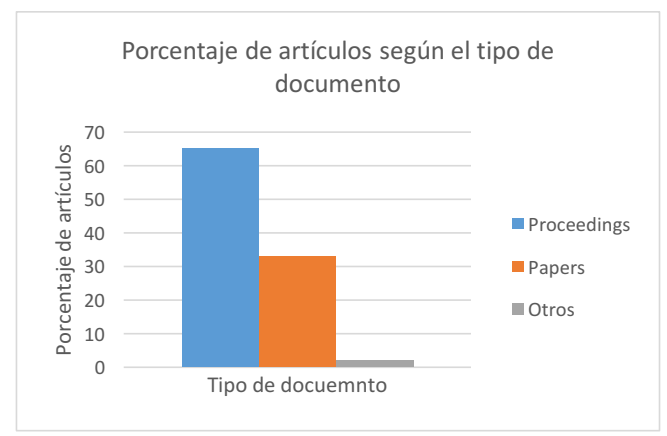

Categorías de la web of Science y áreas de investigación.

Estas dos variables están intrínsecamente relacionadas, ya que las categorías de la Web of Science están condicionadas por las diversas áreas de investigación. Por este motivo existe repetición entre categorías y áreas. En la figura 6 se observa como Education \& Educational research acoge el $83 \%$ de las publicaciones sobre la temática trabajada seguido por la categoría Education Scientific Disciplines con un 6\%. Entre ambas representan el $90 \%$ de las publicaciones.

Figura 6. Porcentaje de artículos por categorías de la Web os Science

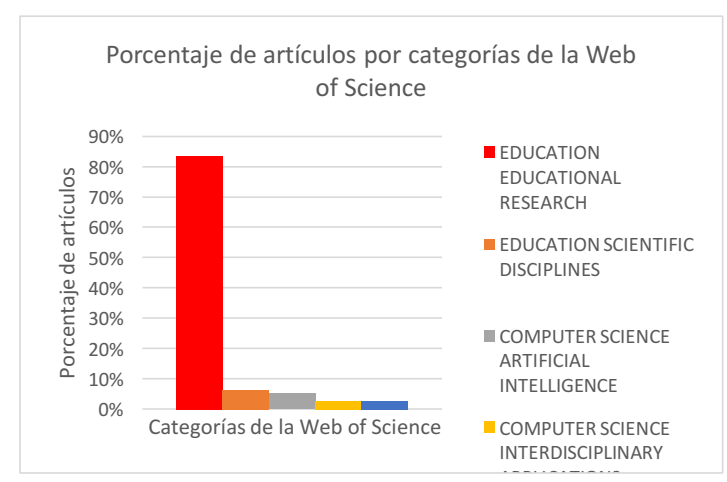

En la figura 7 se observa que las áreas Education \& Educational Research; Psychology acumuIan el $90 \%$ de las investigaciones realizadas. Resulta indicativo que el área de Computer Science tenga el $6 \%$ de los artículos. 
Figura 7. Porcentaje de artículos por áreas de investigación.

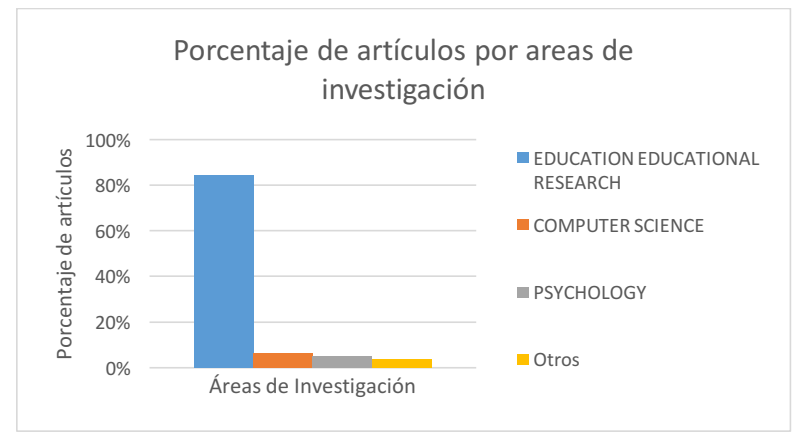

Autores y centros de procedencia.

Otra de los indicadores sobre los que realizar el estudio son aquellos autores que generan una mayor cantidad de artículos y cuál es su centro de procedencia. Esta información queda reflejada en las figuras 7 y 8 . Tras el análisis de la figura 7 se observa que los 5 primeros autores acumulan el $50 \%$ de la producción científica con un $10 \%$ cada uno de ellos. En la figura 8 se observa que el centro de mayor producción de la temática es la Universidad de Granada con un 15\% mientras que la Universidad de Burgos presenta un 12\%.

Figura 8. Porcentaje de artículos por autores

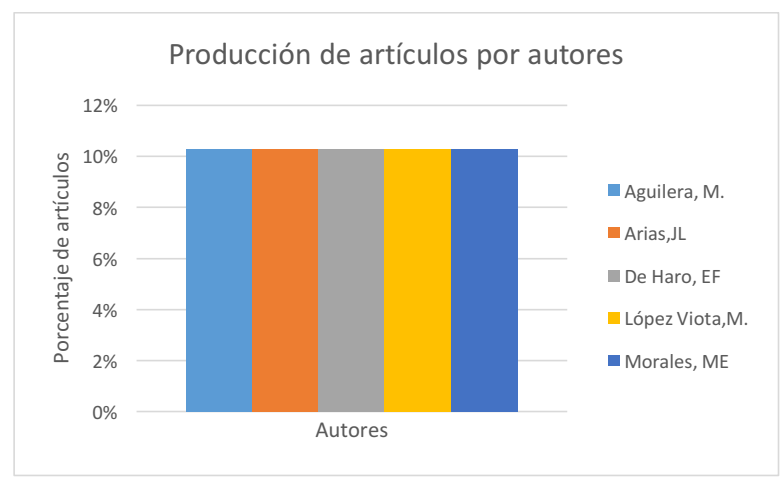


Figura 9. Porcentaje de artículos por procedencia

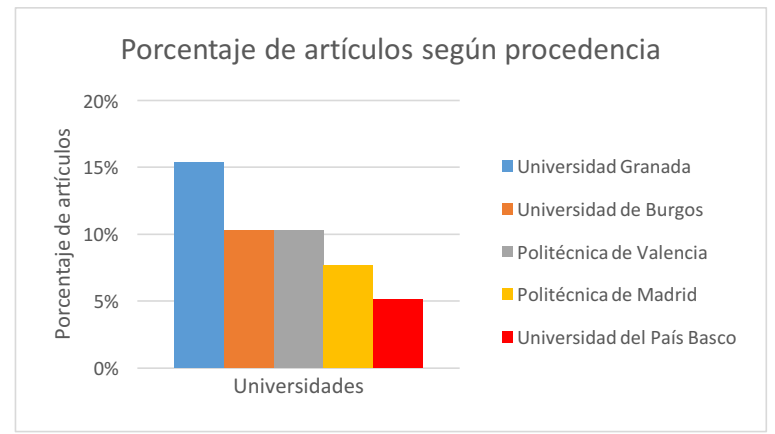

Coautoría.

La última de las variables analizadas es la cantidad de autores que han participado en cada publicación. Esta información queda recogida en la figura 10; el análisis nos indica que la gran mayoría de los trabajos $25 \%$ tienen dos autores como equipo de trabajo, seguida de las autorías individuales con el casi $20 \%$ de las publicaciones. Resulta indicativo que los equipos que oscilan entre 1 y 4 autores acumulan el $80 \%$ de los resultados científicos sobre la temática.

Figura 10. Porcentaje de artículos por autores

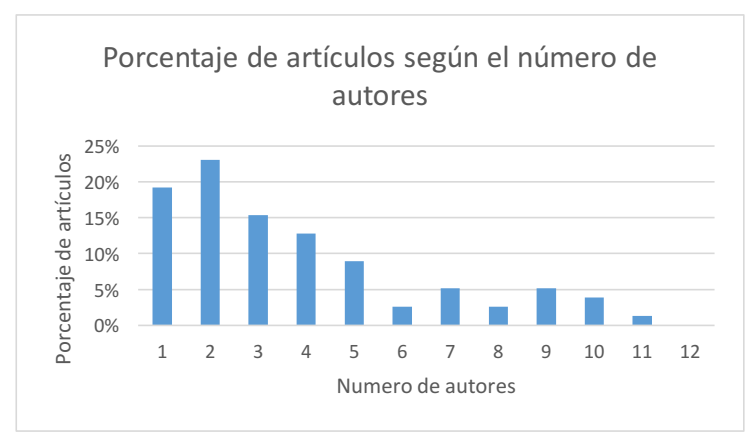

\section{DISCUSIONES}

A lo largo del desarrollo de la presente investigación, se ha pretendido analizar cuál ha sido la producción científica sobre el Plan de Acción tutorial en la Universidad; Ios diversos casos presentados y las ventajas y desventajas presentadas. Para ello se han utilizado una serie de indicadores que ha servido como punto de partida para la obtención de los resultados. Respecto a las dos primeras variables que fueron el número de citas y los año de publicación se ha podido concluir que el año 2015 fue el que acumuló mayor número de citas además del año más prolijo sobre la temática. Este aspecto en cierta medida se ve justificado por la implementación por parte de la gran mayoría de las Universidad de las adaptaciones curriculares para el alumnado con discapacidad y la implementación del PAT adaptado a las necesidades de este alumnado. Por otro lado los artículos publicados en la gran mayoría casi el $70 \%$ son artículos de congresos que muestra que es un campo en continuo desarrollo y mejora. 
En cuanto al idioma de publicación y los países productores, se ha podido observar que el inglés es el idioma dominante, a pesar de ello no existe ningún país de habla inglesa como el mayor productor de artículos. Siendo España la que ocupa la primera posición en esta clasificación. Esta contradicción en cierta medida se debe a que existen muchos países como España, Méjico entre otros que utilizan el inglés como lengua de publicación. Como consecuencia de esta afirmación se observa que existen diversos centros de investigación (Universidad de Granada, Universidad de Burgos) donde se trabaja el PAT en la Universidad y el alumnado con Discapacidad. Por el contrario en países como Méjico existen más países que trabajan la temática pero la producción científica se considera casi despreciable.

Tanto en las categorías de la Web of Science como en la áreas, los resultados indican que Education Educational Research es el área/ categoría más productiva. Lo que indica el cariz meramente educativo que tiene la temática trabajada. A pesar de ello las categorías y áreas tecnológicas obtienen un mayor peso debido a la gran versatilidad que ofrecen las tecnologías para adaptar el PAT a la diversidad del alumnado presenta. Destacan poderosamente las áreas Computer Science y la categoría Computer Science Artificial Intelligence.

En cuanto a las coautorías, se puede observar como los equipos de trabajo son relativamente pequeños lo que indican una baja versatilidad de trabajo a la hora de implementar nuevas medidas. Tiene un gran poder los equipos que tienen entre 1-2 autores; llegando hasta 4 autores como el tamaño ideal para trabajar la temática. Se considera poco productivo el trabajar con equipos de un mayor número.

\section{CONCLUSIONES} llan:

En el presente trabajo se ha obtenido las siguientes conclusiones que a continuación se deta-

El periodo 2015-2016 es donde se produce una mayor cantidad de publicaciones sobre la temática que coincide con el mayor número de citaciones.

EI PAT para el alumnado con discapacidad en la universidad es un campo en desarrollo y como consecuencia de ella son los congresos donde se producen el mayor número de publicaciones.

El inglés es el idioma de publicación a pesar de que España es el mayor productor sobre la temática y de que muchos de los países son de habla hispana y publican en inglés.

La categoría/área de mayor producción en la Web of Science es Education Educational Research lo que corrobora que es un campo meramente educativa a pesar de que la tecnología está avanzando mucho en su aplicación

Los equipos de trabajo para el PAT en el alumnado con discapacidad son relativamente pequeños oscilando entre 1-4 componentes a pesar de que los 1-2 autores tienen también un gran peso. Los equipos de más de 5 autores tienen poco peso.

Como futuras líneas de investigación del trabajo se plantea la posibilidad de poder extender el estudio a otras bases de datos lo que permitiría trabajar con una mayor muestra. Además sería interesante analizar en profundidad cuales son los campos y las temáticas a las cuales ha sido aplicada la realidad aumentada y las posibles y problemas generados.

\section{REFERENCIAS BIBLIOGRÁFICAS}

Álvarez, M., \& Rodríguez, S. (2000). Cambios socioeducativos y orientación en el siglo XXI: Nuevas estructuras, roles y funciones. En AA.VV., Hacia el tercer milenio: Cambios educativos y educación para el cambio (pp. 637- 686). Madrid: Sociedad Española de Pedagogía.

Álvarez, M. (2008). La tutoría académica en el Espacio Europeo de la Educación Superior. Revista Interuniversitaria de Formación del Profesorado, 22(1), 71-88. 
Álvarez González, M. (2009). Planificación y organización de la tutoría en la universidad. En M. Álvarez González y R. Bisquerra (Coords.), Manual de orientación y tutoría. Barcelona: Wolters Kluwer España.

Álvarez, M. (2010). La orientación y la tutoría en la transición Educación Secundaria-Universidad. En M. Álvarez González y R. Bisquerra (Coords.), Manual de orientación y tutoría. Barcelona: Wolters Kluwer España.

Álvarez, M. (2013). Hacia un modelo integral de la tutoría universitaria. En M. Álvarez González y R. Bisquerra (Coords.), Manual de orientación y tutoría. Barcelona: Wolters Kluwer España.

Álvarez M. (2014). La orientación y la tutoría universitaria en la formación de los grados y postgrados. Las transiciones académicas. En P. Figuera (Coord.), Persistir con éxito en la Universidad: De la investigación a la acción. Barcelona: Laertes.

Bricall, J.M. (2000). Informe Universidad 2000. Madrid: CRUE.

García, N. (2008). La función tutorial de la Universidad en el actual contexto de la Educación Superior. Revista Interuniversitaria de Formación del Profesorado, 22(1), 21-28.

Lázaro, Á. (2002). La acción tutorial de la función docente universitaria. En V. Álvarez Rojo y A. Lázaro (Coords.), Calidad de las Universidades y orientación universitaria (pp. 249- 281). Málaga: Aljibe.

Lorenzo, G., Lledó, A., Arráez, G., Lorenzo. A. (2017). La acción tutorial como acompañamiento en el alumnado universitario con discapacidad: Hacia una educación inclusiva. International Journal of Developmental and Educational Psychology, 3(1),137-144.

Moriña, Díez, A. y Molina- Romo, V. (2011). La universidad a análisis: las voces del alumnado con discapacidad. Revista de Enseñanza Universitaria, 37(1), 23-35.

Lledó, A; Álvarez, JD; Grau, S; González, C; Roig, R; Lorenzo, G; \& Tortosa, MT. (2008). Academia learning and achievement The mentoring program in the university as a measure of quality the learning students's process: A proposal for action at the university of Alicante. In proceedings of XXIX. Congress of Psychology. Berlin. Alemania.

Lledó, A. (2015). La inclusión educativa de la discapacidad en la universidad; una cuestión pendiente para seguir avanzando en Educación Superior. En Lucía Herrera (coord.) Retos y desafíos de la Educación Superior desde la perspectiva del profesorado universitario. Madrid:Síntesis.

Perandones, T.M. \& Lledó, A. (2009) La función del profesorado universitario como tutor. Experiencias en el Programa de Acción Tutorial en la Universidad de Alicante. En Roig, R.; Blasco, J.; Gilar, R.; Lledó, A.; Mañas, C.; (eds.). Investigar desde un contexto educativo innovador. Alcoy: Marfil.

Pérez (2006). Tutorías. En M. de Miguel (Coord.), Metodologías de enseñanza y aprendizaje para el desarrollo de competencias (pp. 133- 168). Madrid: Alianza Editorial.

Rodríguez, S (2004). Manual de tutoría universitaria. Barcelona: Octaedro/ICE-UB.

Rodríguez, S. (2006). Las seis caras de la función tutorial. En M. Álvarez González (Coord.), Projecte de formació per a la tutoria universitaria en el marc de l'Espai Europeu d'Educació Superior a la Universitat de Barcelona. Barcelona: Agència de Gestió d’Ajuts Universitaris i de Recerca de la Universitat de Barcelona. 
